\title{
DETERMINATION OF REACTIVITY AND THERMODYNAMIC ANALYSIS OF SOLID COPPER-BASED OXYGEN CARRIERS FOR CHEMICAL RECIRCULATION
}

\author{
Adolfo L. Figueredo ${ }^{1 *}$, Fabiola F. Carvalho ${ }^{2}$, Jose C Nascimento ${ }^{2}$, Juan A.C. Ruiz ${ }^{2}$, \\ Osvaldo Chiavone-Filho ${ }^{1}$, Camila G. Pereira ${ }^{1}$
}

${ }^{1}$ Department of Chemical Engineering, NUPEG, Federal University of Rio Grande do Norte, UFRN, Av. Senador Salgado Filho, 3000, ZIP 59078-970, Natal-RN, Brazil.

${ }^{2}$ Laboratory of Sustainability, Senai Institute of Innovation in Renewable Energy, Av. Capitão Mor Gouveia, 2770, ZIP 59063-400, Natal-RN, Brazil.

*e-mail: adolfofigueredo@outlook.com

\section{Supporting Information}

In the supporting information contains tables $\mathrm{S} 1$ to $\mathrm{S} 3$, corresponding to:

Table S1: Kinetic models used to verify the adequacy of experimental data;

Table S2: Reduction temperatures of each event and $\mathrm{H}_{2}$ consumption of OCs at each event during TPR analysis.

Table S3: Kinetic parameters of the redox reaction of copper-based OCs at $1123 \mathrm{~K}$ using ethanol as fuel.

Table S1. Kinetic models.

\begin{tabular}{lcc}
\hline \multicolumn{1}{c}{ Kinetic Models } & $f\left(X_{S}\right)$ & $g(x)$ \\
\hline Shrinking core ${ }^{\mathrm{a}}$ & $2\left(1-X_{S}\right)^{1 / 2}$ & $1-\left(1-X_{S}\right)^{1 / 2}$ \\
Nucleation & $m\left(1-X_{S}\right)\left[-\ln \left(1-X_{S}\right)\right]^{1-1 / m}$ & {$\left[-\ln \left(1-X_{S}\right)\right]^{1 / m}$} \\
Three-dimensional diffusion & $\frac{m}{2}\left(1-X_{S}\right)^{2 / 3}\left[1-\left(1-X_{S}\right)^{1 / 3}\right]$ & {$\left[1-\left(1-X_{S}\right)^{1 / 3}\right]^{2}$} \\
& \\
\hline ahase-boundary controlled (contracting cylinder)
\end{tabular}


where $m$ is the kinetic exponential factor in the nucleation model, which depends in part on the nucleation frequency and describes the rate where the mechanism develops itself (grain growth).

Table S2. Reduction temperatures of each event and $\mathrm{H}_{2}$ consumption of OCs at each event during TPR analysis.

\begin{tabular}{ccccccc}
\hline \multirow{2}{*}{ Events } & $\begin{array}{c}\text { Temperature } \\
\text { range }\left({ }^{\circ} \mathrm{C}\right)\end{array}$ & \multicolumn{3}{c}{ Consumption of $\mathrm{H}_{2}\left(\mathrm{mmolg}^{-1}\right)$} & Reduced \\
\cline { 3 - 5 } & $<205$ & $05 \mathrm{Cu}-\mathrm{R}$ & $10 \mathrm{Cu}-\mathrm{R}$ & $15 \mathrm{Cu}-\mathrm{R}$ & $10 \mathrm{Cu} / \mathrm{Ce}-\mathrm{R}$ & species \\
\hline 1 & $205-230$ & - & 0.718 & 1.054 & - & $\mathrm{Cu}^{+1}$ \\
2 & $350-430$ & 0.094 & 0.127 & 0.239 & 0.464 & $\mathrm{CuAl}_{2} \mathrm{O}_{4}$ \\
3 & $550-555$ & 0.097 & 0.123 & 0.319 & 0.131 & $\mathrm{CuAl}_{2} \mathrm{O}_{4}$ \\
4 & $730-760$ & 0.116 & 0.063 & 0.159 & 0.174 & $\mathrm{CuAl}_{2} \mathrm{O}_{4}$ \\
\hline 5 & & & & & & \\
\hline
\end{tabular}

Table S3. Kinetic parameters of the redox reaction of copper-based OCs using ethanol as fuel.

\begin{tabular}{cccccccc}
\hline \multirow{2}{*}{ OC } & $m$ & \multicolumn{2}{c}{ Nucleation model } & \multicolumn{2}{c}{ Shrinking-core model } & \multicolumn{2}{c}{ Diffusion Model } \\
\cline { 3 - 8 } & & $k$ & $\mathrm{R}^{2}$ & $k$ & $\mathrm{R}^{2}$ & $k$ & $\mathrm{R}^{2}$ \\
\hline $05 \mathrm{Cu}-\mathrm{R}$ & 1.373 & 0.300 & 0.995 & 0.116 & 0.987 & 0.225 & 0.994 \\
$10 \mathrm{Cu}-\mathrm{R}$ & 1.502 & 0.173 & 0.991 & 0.066 & 0.966 & 0.124 & 0.978 \\
$15 \mathrm{Cu}-\mathrm{R}$ & 1.609 & 0.128 & 0.988 & 0.048 & 0.931 & 0.088 & 0.947 \\
$10 \mathrm{Cu} / \mathrm{Ce}-\mathrm{R}$ & 1.677 & 0.131 & 0.987 & 0.049 & 0.920 & 0.088 & 0.982
\end{tabular}

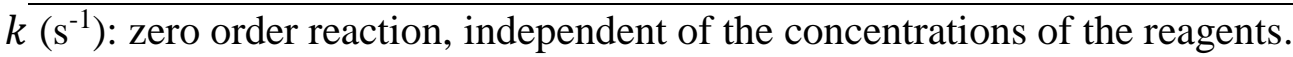

